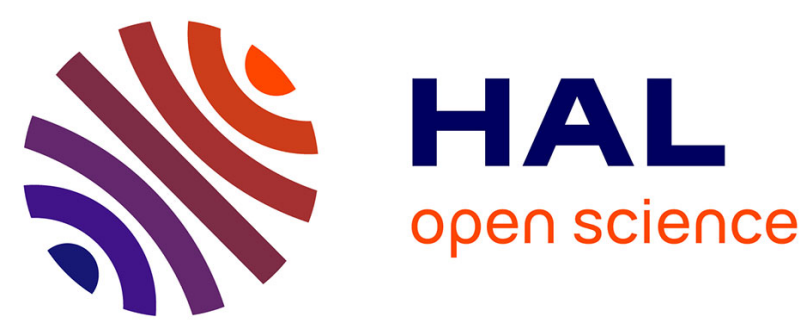

\title{
Automatic Tip-Steering of Concentric Tube Robots in the Trachea Based on Visual SLAM
}

Cédric Girerd, Andrey Kudryavtsev, Patrick Rougeot, Pierre Renaud, Kanty Rabenorosoa, Brahim Tamadazte

\section{- To cite this version:}

Cédric Girerd, Andrey Kudryavtsev, Patrick Rougeot, Pierre Renaud, Kanty Rabenorosoa, et al.. Automatic Tip-Steering of Concentric Tube Robots in the Trachea Based on Visual SLAM. IEEE Transactions on Medical Robotics and Bionics, 2020, 2 (4), pp.582 - 585. 10.1109/TMRB.2020.3034720 . hal-03053017

\section{HAL Id: hal-03053017 https://hal.science/hal-03053017}

Submitted on 10 Dec 2020

HAL is a multi-disciplinary open access archive for the deposit and dissemination of scientific research documents, whether they are published or not. The documents may come from teaching and research institutions in France or abroad, or from public or private research centers.
L'archive ouverte pluridisciplinaire HAL, est destinée au dépôt et à la diffusion de documents scientifiques de niveau recherche, publiés ou non, émanant des établissements d'enseignement et de recherche français ou étrangers, des laboratoires publics ou privés. 
See discussions, stats, and author profiles for this publication at: https://www.researchgate.net/publication/344895231

\section{Automatic Tip-Steering of Concentric Tube Robots in the Trachea Based on Visual SLAM}

Preprint $\cdot$ October 2020

DOI: 10.1109/TMRB.2020.3034720

CITATIONS

6 authors, including:

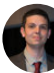

Cédric Girerd

University of California, San Diego

10 PUBLICATIONS 21 CITATIONS

SEE PROFILE

8. Patrick Rougeot

1 École Nationale Supérieure de Mécanique et des Microtechniques

55 PUBLICATIONS 576 CITATIONS

SEE PROFILE

Some of the authors of this publication are also working on these related projects:

Project $\mu$ RoCS: MicroRobot-assisted Cholesteatoma Surgery View project

Project NEMRO: Impact of Smell Deficiency on Neurodegenerative Diseases View project
READS

27

Andrey V. Kudryavtsev

14 PUBLICATIONS 109 CITATIONS

SEE PROFILE

Kanty Rabenorosoa

UBFC

69 PUBLICATIONS 712 CITATIONS

SEE PROFILE 


\title{
Automatic Tip-Steering of Concentric Tube Robots in the Trachea Based on Visual SLAM
}

\author{
Cédric Girerd $^{1,2 *}$, Andrey V. Kudryavtsev ${ }^{1 *}$, Patrick Rougeot ${ }^{1}$, Pierre Renaud ${ }^{3}$, Kanty Rabenorosoa ${ }^{1}$ \\ and Brahim Tamadazte ${ }^{1,4}$
}

\begin{abstract}
Concentric tube robots are continuum robots particularly well-suited for minimally-invasive surgery. Their small diameter, natural free channel and snake-like shape unlock the potential for applications that require a high degree of dexterity in confined spaces. The synthesis and planning of such robots are generally based on the patient's anatomy and the type of surgery to be performed. However, their practical deployment can be challenging. Open-loop approaches are prone to errors due to modeling accuracy or physiological motions of the patient during the procedure, and human-in-the-loop approaches require specific skills from the surgeon. In this paper, we propose an automatic tip-steering approach for concentric tube robots based on visual SLAM (simultaneous localization and mapping). This allows a closed-loop control scheme to compensate for open-loop errors, and provides to the surgeon the opportunity to focus on the medical procedure, simplifying the management of the deployment. A method is proposed in the case of a robot geometry that achieves approximate follow-the-leader deployment. It is validated by performing an automatic tip-steering task in the trachea using a realistic simulator for concentric tube robots.
\end{abstract}

Index Terms-Medical robotics, concentric tube robots, tip steering, visual SLAM.

\section{INTRODUCTION}

$\mathbf{T}$ ASK automation is an interesting area in medical robotics. In particular, the automation of intracorporeal deployment tasks in a time-varying and patient-specific environment is an exciting, yet open research topic. In this paper, we focus on patient-specific automatic deployment of concentric tube robots (CTRs). CTRs are a particular type of continuum robots [1] made of precurved, nested tubes that interact in bending and torsion, defining the robot backbone shape. The latter can be changed by relative rotations and translations of the tubes. The shape variety of a CTR is limited by the initial geometry of its tubes, whose synthesis is based on surgical task requirements and patient's anatomy. While CTR tube design can take patient inter-variability into account, the shape of the robot still has to be adjusted by modification of its joint variables when used on a specific patient. This is usually performed offline during a planning task that requires 3-D preoperative images (e.g., computerized tomography scan) of the patient [2]. A first approach based on point clouds has been proposed [3], which can adapt to changes of geometry of

\footnotetext{
${ }^{1}$ Authors are with FEMTO-ST Institute, Univ. Bourgogne FrancheComté, CNRS, Besançon, France.

2 Author is with the Department of Mechanical and Aerospace Engineering, University of California, San Diego, La Jolla, CA 92093 USA cgirerdeeng. ucsd.edu (corresponding author)

${ }^{3}$ Author is with ICube, UDS-CNRS-INSA, Strasbourg, France.

${ }^{4}$ Author is with Sorbonne Université, CNRS UMR 7222, INSERM U1150, ISIR, F-75005, Paris, France.

* These authors contributed equally to this work.
}

the anatomy during the planning, and has the potential to be used online. To lower the duration and cost of the procedure, we presented an approach that allowed online tip-steering of a CTR using monocular visual SLAM (Simultaneous Localization And Mapping) [4]. The focus was put on the tip of the robot, for which obstacles were avoided, while the rest of the body had an approximate follow-the-leader (FTL) deployment, thanks to tube geometries that allow such behavior. In this paper, we now investigate the use of this approach in our opensource simulator (see https://avkudr.github.io/visa-model-ctr) with tip-steering of a CTR in a realistic model of trachea, for applications such as vocal cords surgery [5] or laryngeal surgery [7], that motivate this work.

\section{MATERIALS AND METHODS}

\section{A. Robot geometry}

The proposed method first requires tube geometries that strictly respect FTL design rules [8], which are then deployed in an approximate FTL manner, using a tube actuation sequence computed online during the tip-steering task. Given the planar nature of the buccal cavity that leads to the trachea, the case of a planar robot made out of constant-curvature tubes arranged in a plane, with either aligned or opposite curvatures, is considered. This constrains the tube angles, that are not actuated, as opposed to their translation, that allow to change the orientation of the CTR tip in its deployment plane. One

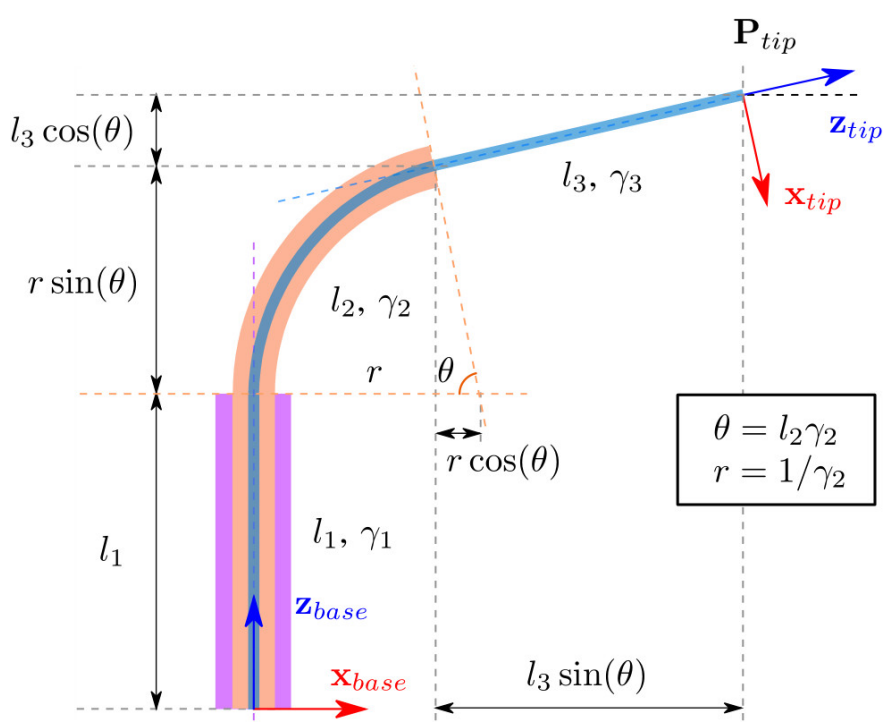

Fig. 1. Geometry of the CTR under consideration. $l_{i}$ and $\gamma_{i}$ are the length and curvature of $i$-th CTR link. 
possible access path to the trachea is composed of successive straight, curved and straight sections. A CTR made out of 3 tubes is thus selected, that form a robot that has a straight, curved, and straight link. It is visible in Fig. 1, along with relevant parameters.

Let $\mathbf{q}$ be the set of coordinates defining the deployed lengths of the CTR tubes with respect to the base frame. In the example of Fig. 1, with tubes 1, 2 and 3 represented in blue, orange and violet, with tube 1 being the innermost tube, $\mathbf{q}^{\top}=\left(\begin{array}{lll}l_{1}+l_{2}+l_{3} & l_{1}+l_{2} & l_{1}\end{array}\right)$, with $l_{i}$ the length of the CTR links, with link 1 being the most proximal link. The tube deployment sequence is obtained by a control algorithm that relies on a 3-D reconstruction of the environment, using a visual SLAM approach. For this purpose, DSO-SLAM [9] is selected for its ability to compute both the localization of the camera and the sparse mapping of the surrounding environment directly using photometric information instead of geometric ones (e.g., key-points, descriptors). This makes it a suitable and robust approach in the case of low-textured environments as is the case for medical applications. A control law that uses the information provided by the DSO-SLAM algorithm is detailed in the following for tip-steering purposes.

\section{B. Tip-steering task}

Automatic tip steering is achieved by combining joint velocities computed from two distinct tasks: the "Translation" (T) and "Orientation" (O) tasks. The functions that represent these tasks, $\mathbf{q}_{\mathbf{T}}$ and $\mathbf{q}_{\mathbf{O}}$, respectively, are associated to the fixed gains $\lambda_{T}$ and $\lambda_{O}$, respectively, as well as the control gains $\eta$ and $(1-\eta)$, respectively, that evolve during deployment, and depend on the presence of obstacles close to the CTR tip. The complete control law is given by Eq. (1). Each task is defined in the following.

$$
\dot{\mathbf{q}}=\left(\begin{array}{c}
\dot{l_{1}}+\dot{l_{2}}+\dot{l_{3}} \\
\dot{l_{1}}+\dot{l_{2}} \\
\dot{l_{1}}
\end{array}\right)=\underbrace{\lambda_{T} \eta \dot{\mathbf{q}}_{\mathbf{T}}}_{\text {Translation }}+\underbrace{\lambda_{O}(1-\eta) \dot{\mathbf{q}} \mathbf{O}}_{\text {Orientation }}
$$

1) Translation task: The primary task of the robot is to progress inside the anatomical area, by moving its tip forward. This corresponds to a translation motion of the inner straight tube of the CTR, using Eq. (2), with $v$ the deployment speed of the inner tube.

$$
\dot{\mathbf{q}}_{\mathbf{T}}=\left(\begin{array}{l}
v \\
0 \\
0
\end{array}\right)
$$

2) Orientation task: When facing an obstacle, the "Orientation" task is activated, during which the CTR rotates about its tip at a velocity $\omega$ in order to change its tip insertion angle. This task is formulated in Eq. (3):

$$
\dot{\mathbf{q}}_{\mathbf{O}}=\mathbf{J}_{\mathbf{S}}^{\mathbf{i n v}} \mathbf{J}_{\mathbf{O}}^{\text {inv }}\left(\begin{array}{l}
0 \\
0 \\
\omega
\end{array}\right),
$$

which involves the Jacobian matrix $\mathbf{J}_{\mathbf{O}}^{\text {inv }}$, given by Eq. (4), that represents the relationship between the CTR tip pose, i.e., translation and orientation of the tip in the plane, and the deployed link lengths. The matrix $\mathbf{J}_{\mathbf{O}}^{\mathbf{i n v}}$ is obtained as follows:

$$
\mathbf{J}_{\mathbf{O}}^{\mathbf{i n v}}=\left(\begin{array}{ccc}
\frac{\partial x_{E} E}{\partial l_{1}} & \frac{\partial x_{E E}}{\partial l_{2}} & \frac{\partial x_{E E}}{\partial l_{3}} \\
\frac{\partial z_{E}}{\partial l_{1}} & \frac{\partial z_{E E}}{\partial l_{2}} & \frac{\partial z_{E E}}{\partial l_{3}} \\
\frac{\partial \theta}{\partial l_{1}} & \frac{\partial \theta}{\partial l_{2}} & \frac{\partial \theta}{\partial l_{3}}
\end{array}\right)^{-1}
$$

and the inverse of the so-called Specific Jacobian matrix $\mathbf{J}_{\mathbf{S}}^{\text {inv }}$, given by Eq. (5), expresses the relationship between the link lengths and the tube lengths.

$$
\mathbf{J}_{\mathbf{S}}^{\mathbf{i n v}}=\left(\begin{array}{lll}
1 & 1 & 1 \\
1 & 1 & 0 \\
1 & 0 & 0
\end{array}\right)
$$

3) Combining the tasks: The balance between the "Translation" and "Orientation" tasks depends on the presence of obstacles close to the CTR tip. Only smooth deviations of the environment walls are considered, with no frontal obstacles faced at the tip. It is based on a repulsive force $F$, computed as follows. First, a point $\mathbf{P}_{t i p}^{*}=\mathbf{P}_{t i p}+d_{\pi} \mathbf{z}_{t i p}$ is created, as well as a plane $\boldsymbol{\pi}$, normal to $\mathbf{z}_{t i p}$, that contains $\mathbf{P}_{\text {tip }}^{*}$ (see Fig. 2). All the 3D reconstructed points given by the SLAM algorithm that are at a distance lower than $d_{\pi}^{*}$ to $\pi$, and closer than a distance $d_{\mathcal{X}}^{m i n}$ to the $\left(\mathbf{P}_{t i p}, \mathbf{P}_{t i p}^{*}\right)$ axis of the camera, are projected on the plane $\pi$. A force $F_{k}$ is computed during the control iteration $k$, which is inversely proportional to the square of their distances to $\mathbf{P}_{t i p}^{*}$, so that they repel the tip of the robot. As the CTR has a planar shape and obstacles are avoided on its deployment plane, only the component of $F_{k}$ along $\mathrm{x}_{t i p}$ is considered. The 3D point cloud given by the SLAM algorithm can be subject to image noise, so $F_{k}$ is filtered over time using the expression given in Eq. (6), with $\delta$ a weighting filtering coefficient.

$$
F=\delta F_{k-1}+(1-\delta) F_{k}
$$

The obtained filtered repulsive force $F$ is finally used to compute $\eta$ (see Eq. (7)), required to compute the gains $\eta$ and $(1-\eta)$ of the "Translation" and "Orientation" tasks, respectively (see Eq. (17). In Eq. (7), the scalar $a$ controls the slope of $\eta$. As $\eta$ lies in the interval $[0.5,1]$, the "Translation" task, weighted with the gain $\eta$, is always activated during the

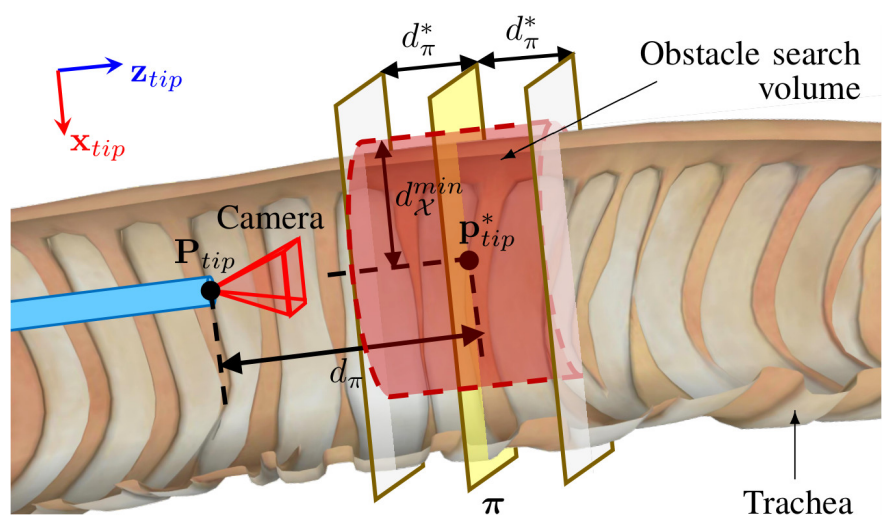

Fig. 2. The points of $\mathcal{H}$ that are closer than $d_{\pi}^{*}$ to the plane $\pi$ are projected on it. The resulting set of $2 \mathrm{D}$ points $\mathcal{X}$ is used for motion planning. 
CTR control, while the "Orientation" task, weighted with the gain $(1-\eta)$, is only activated in the presence of obstacles.

$$
\eta=1.5-\frac{1}{1+e^{-a|F|}}
$$

Finally, we choose an angular velocity $\omega=\operatorname{sign}(F)$ for the "Orientation" task, allowing the CTR tip to be oriented in a direction that depends on the location of the obstacle faced. Furthermore, since CTRs can be subject to instabilities, a local stability criterion is implemented, with the condition $\operatorname{det}\left(\boldsymbol{W}_{2}\right)>0$ that must hold during deployment, with $\boldsymbol{W}_{2}$ which depends on the tube design parameters and deployed lengths [10]. The stability of the CTR is first assessed offline for sampled deployed tube lengths that enable the area of interest to be reached. It is then evaluated during the deployment task as a safety measure, as it is a critical condition for the CTR body to remain in its plane of deployment. The deployment is stopped if the CTR approaches instabilities, i.e. if $\operatorname{det}\left(\boldsymbol{W}_{2}\right)<\epsilon$, with $\epsilon>0$ a stability margin.

\section{RESULTS}

The proposed control method is assessed using an inhouse simulator [11]. It contains an anatomical model of the trachea, the CTR, and a virtual camera attached to its tip. The characteristics of the tubes used for the simulation are reported in Table I They allow to form a straight $\left(0.00 \mathrm{~mm}^{-1}\right)$, curved $\left(0.02 \mathrm{~mm}^{-1}\right)$ and straight $\left(0.00 \mathrm{~mm}^{-1}\right)$ links, with a curved value that is well-adapted to the considered anatomical area. As the robot tubes do not undergo any torsion during deployment, a torsionally-rigid kinematic CTR model [1] was implemented in the simulator. The parameters selected for the simulation are $\lambda_{T}=1, \lambda_{O}=1, v=0.25 \mathrm{~mm} . \mathrm{s}^{-1}, d_{\pi}=3 \mathrm{~mm}$, $d_{\pi}^{*}=0.4 \mathrm{~mm}, d_{\mathcal{X}}^{\min }=2 \mathrm{~mm}, \delta=0.95, a=10$ and $\epsilon=0.1$. The simulation starts with the tubes partially deployed, with the CTR tip at the entry of the throat, as this region does not present any deployment challenge.

Fig. 3 represents the 3-D reconstruction of the trachea by the SLAM algorithm. It illustrates the obtained reconstructed shape at the end of the deployment (see the provided video for the reconstructed shape of the trachea during the entire deployment). As visible in this figure, the robot successfully deploys inside the trachea, while reconstructing it in $3 \mathrm{D}$. The bottom-left and right images depict the camera view in the represented pose, with and without the detected features. In a complementary way, Fig. 4 is a planar representation of the reconstructed trachea, that corresponds to a projection of the detected keypoints on the CTR deployment plane. It is represented at different stages of the deployment, with the red lines representing the camera path, the blue points representing the earliest keypoints detected by the camera, and the yellow ones representing the latest ones.

Fig. 5a represents the values of $\eta$ and $F$ during deployment, and Fig. $5 b$ represents the deployed lengths of the tubes over time. Three different steps can be observed during deployment, that are labeled from 1 to 3 in these figures. During the first

TABLE I

CHARACTERISTICS OF THE TUBES USED FOR THE SIMULATION OF A DEPLOYMENT INSIDE THE TRACHEA.

\begin{tabular}{lccc}
\hline Tube index & 1 & 2 & 3 \\
\hline Material & Nitinol & Nitinol & Steel \\
Young Modulus $(\mathrm{GPa})$ & 80 & 80 & 220 \\
Poisson's ratio & 0.33 & 0.33 & 0.30 \\
Inner diameter $(\mathrm{mm})$ & 0.610 & 0.770 & 1.100 \\
Outer diameter $(\mathrm{mm})$ & 0.650 & 1.010 & 1.600 \\
Curvature $\left(\mathrm{mm}^{-1}\right)$ & 0.0000 & 0.0423 & 0.0023 \\
Total length $(\mathrm{mm})$ & 300.0 & 143.0 & 60.0 \\
\hline
\end{tabular}

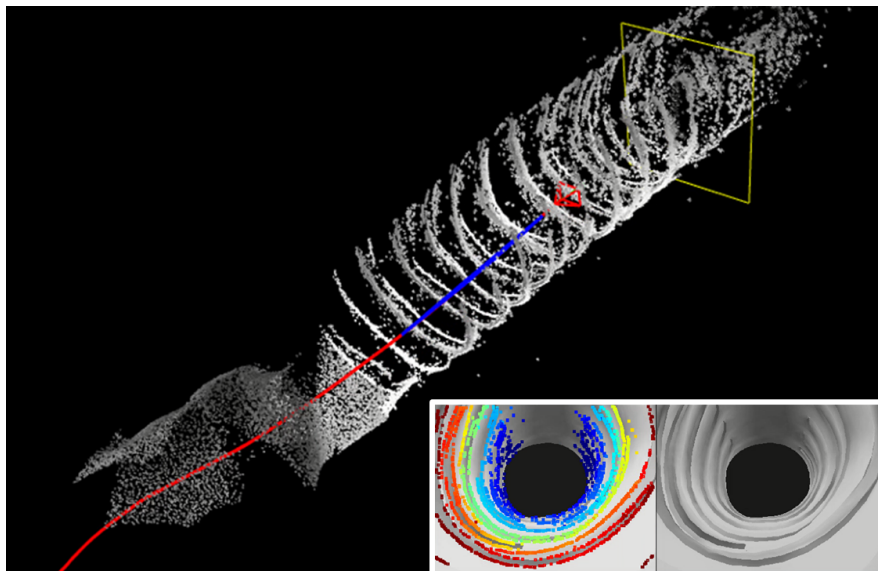

Fig. 3. Top: reconstructed 3-D shape of the environment given by the DSOSLAM algorithm, with the red lines showing the camera trajectory. Bottom: frame obtained by the virtual camera during deployment, with and without the detected keypoints.

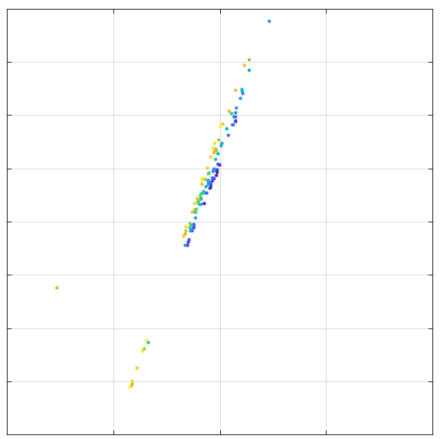

(a) Frame $1 / 1602$

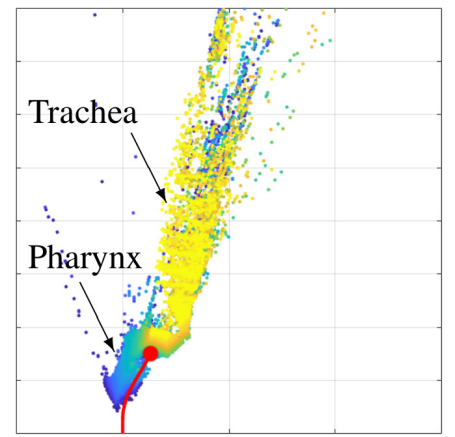

(b) Frame 534/1602

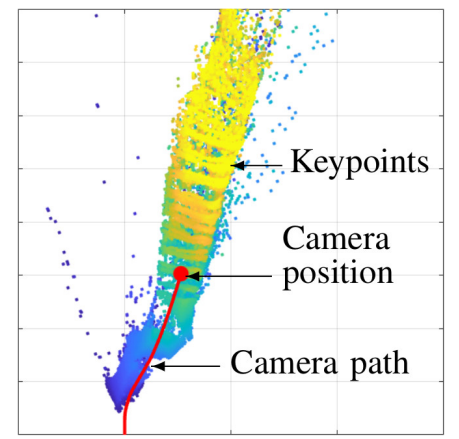

(c) Frame 1060/1602

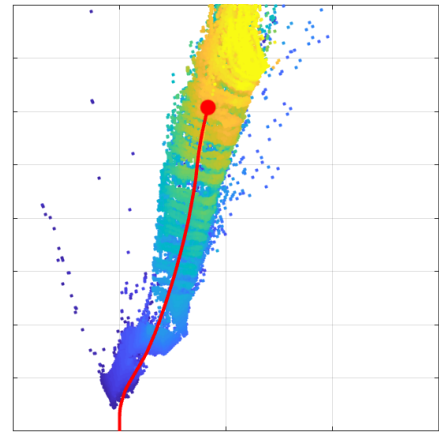

(d) Frame 1600/1602

Fig. 4. Illustration of different steps of the deployment, with the projection of the obtained keypoints on the CTR plane. For a given deployment step, the blue points correspond to the first keypoints detected since the beginning of the deployment, while the yellow ones correspond to the most recent ones. 


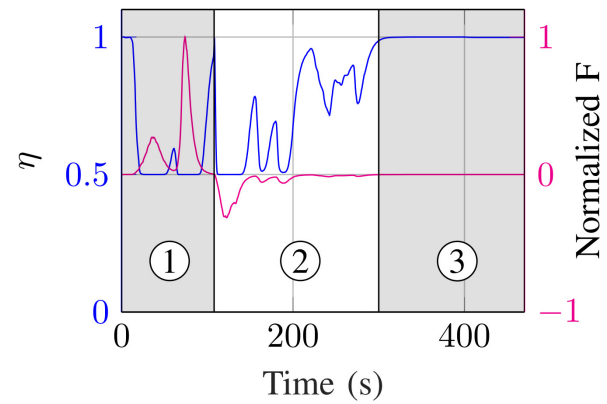

(a)

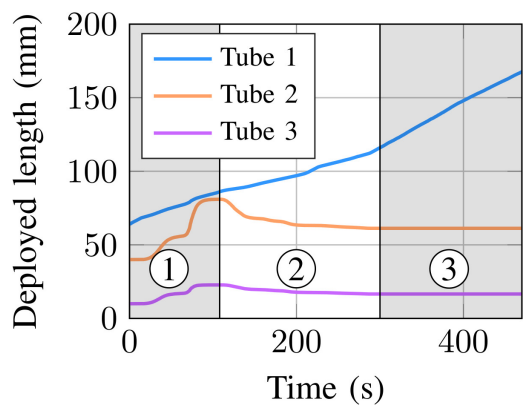

(b)

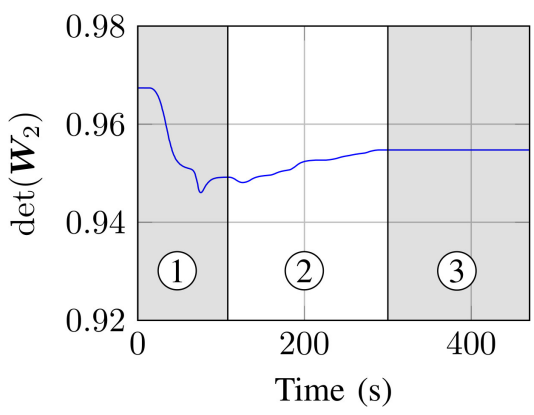

(c)

Fig. 5. (a) normalized repulsive force $F$ (filtered) and $\eta$,(b) deployed tube lengths, and (c) stability of the CTR over time, with the deployment steps identified and labeled, during deployment in the trachea.

one, $F$ is positive, and all the tubes are moving forward. Indeed, the robot first faces the external wall of the throat at the top in the camera frame, which forces its tip to rotate in one direction and increase the length of its curved link. During the second step, $F$ becomes negative, and tubes 2 and 3 are moving backwards. This corresponds to the CTR tip facing the wall of the trachea at the bottom in the camera frame, which forces the tip to rotate in the opposite direction, in order to decrease the length of its curved link. This phenomenon of the CTR tip rotating in two directions in steps 1 and 2 can also be observed in Fig. 4 and in the top image of Fig. 3 , with the red line representing the camera path that has an "S"-like shape. It is also visible in the provided video. Then, during the last deployment step, $F$ equals zero, and only the "Translation" task is active, with the inner tube moving forward, while tubes 2 and 3 remain still. The deployment inside the considered anatomical area was fully conducted and was successful, with no instability encountered by the robot, as visible in Fig. 5c.

Finally, the impact of robot pose errors on the deployment is evaluated. The tracking accuracy of commercial systems was assessed in [12], with reported errors in the order of magnitude of $1 \mathrm{~mm}$ and 1 degree in the pose estimation of the device. We thus generated Gaussian distributions of errors centered on 0 , with $6 \sigma=1 \mathrm{~mm}$ and 1 degree for the translation and rotation pose errors, respectively, and truncated these distributions at $\pm 3 \sigma$. We then performed 30 independent deployment simulations, injecting pose errors on the device frame $\mathcal{R}_{\text {device }}$, defined as the translation of the base frame $\mathcal{R}_{\text {base }}$ (see Fig. 1) by $-100 \mathrm{~mm}$ along the $z$-axis. Despite the pose errors added to the initial unoptimized robot pose, all 30 deployments inside the trachea were successful, which tends to confirm the overall interest of the proposed approach.

\section{CONCLUSION}

In this paper, our visual SLAM-based tip-steering method was presented and implemented in a practical case, for deployment in the trachea. It allows an approximate FTL deployment of a CTR, using a control algorithm that combines a tip translation and orientation task. The tasks are weighted based on the observations of an eye-in-hand camera, located at the tip of the robot. The performance of our approach was evaluated in our in-house simulator, with successful deployments, even in the presence of pose errors. Future work will be focused on experimental evaluations in a realistic anatomical model, collision assessment along the entire robot body, extension of the proposed method to CTRs with tubes that don't strictly respect FTL design rules, and application to other types of continuum robots.

\section{ACKNOWLEDGMENT}

This work was supported by the Equipex ROBOTEX project (ANR-10-EQPX-44-01), the EIPHI Graduate School (ANR17-EURE-0002) and ANR NEMRO (ANR-14-CE17-0013).

\section{REFERENCES}

[1] P. E. Dupont, J. Lock, B. Itkowitz, and E. Butler, "Design and control of concentric-tube robots," IEEE Trans. on Rob., vol. 26, no. 2, pp. 209-225, 2010.

[2] K. Leibrandt, C. Bergeles, and G.-Z. Yang, "Concentric tube robots: Rapid, stable path-planning and guidance for surgical use," IEEE Robotics \& Automation Magazine, vol. 24, no. 2, pp. 42-53, 2017.

[3] A. Kuntz, M. Fu, and R. Alterovitz, "Planning high-quality motions for concentric tube robots in point clouds via parallel sampling and optimization," in 2019 IEEE/RSJ International Conference on Intelligent Robots and Systems (IROS), 2019, pp. 2205-2212.

[4] C. Girerd, A. V. Kudryavtsev, P. Rougeot, et al., "SLAM-based followthe-leader deployment of concentric tube robots," IEEE Rob. and Auto. Lett., pp. 548-555, 2020.

[5] R. Renevier, B. Tamadazte, K. Rabenorosoa, et al. , "Endoscopic laser surgery: Design, modeling, and control," IEEE/ASME Trans. on Mech., vol. 22, no. 1, pp. 99-106, 2017.

[6] D. T. Friedrich, V. Modes, T. K. Hoffmann, et al., "Teleoperated tubular continuum robots for transoral surgery-feasibility in a porcine larynx model," Int. J. Med. Robot Comp. Ass. Surg. , vol. 14, no. 5, p. e1928, 2018.

[7] D. T. Friedrich, V. Modes, T. K. Hoffmann, J. Greve, P. Schuler, and J. Burgner-Kahrs, "Teleoperated tubular continuum robots for transoral surgery-feasibility in a porcine larynx model," The International Journal of Medical Robotics and Computer Assisted Surgery, vol. 14, no. 5, p. e1928, 2018.

[8] H. B. Gilbert, J. Neimat, and R. J. Webster, "Concentric tube robots as steerable needles: Achieving follow-the-leader deployment," IEEE Trans. on Rob., vol. 31, no. 2, pp. 246-258, 2015.

[9] J. Engel, V. Koltun, and D. Cremers, "Direct sparse odometry," IEEE Trans. Pattern Anal. Mach. Intell., vol. 40, no. 3, pp. 611-625, 2017.

[10] H. B. Gilbert, R. J. Hendrick, and R. J. Webster III, "Elastic stability of concentric tube robots: A stability measure and design test," IEEE Trans. on Rob., vol. 32, no. 1, pp. 20-35, 2016.

[11] A. V. Kudryavtsev, K. Rabenorosoa, and B. Tamadazte, "Towards a generic simulator for continuum robot control," in Workshop "Continuum Soft Robotics" at IEEE/RSJ IROS'2018, Oct. 2018.

[12] T. Ameler, K. Blohme, L. Brandt, et al., "A comparative evaluation of steamvr tracking and the optitrack system for medical device tracking," in IEEE Int. Conf. of Eng. in Med. and Bio. Soc., 2019, pp. 1465-1470. 